University of Nebraska - Lincoln

DigitalCommons@University of Nebraska - Lincoln

Industrial and Management Systems

Engineering Faculty Publications

Industrial and Management Systems

Engineering

2000

Digital Human Models for Ergonomics

Jeffrey C. Woldstad

University of Nebraska-Lincoln, Jeffrey.Woldstad@sdsmt.edu

Follow this and additional works at: https://digitalcommons.unl.edu/imsefacpub

Part of the Operations Research, Systems Engineering and Industrial Engineering Commons

Woldstad, Jeffrey C., "Digital Human Models for Ergonomics" (2000). Industrial and Management Systems Engineering Faculty Publications. 10.

https://digitalcommons.unl.edu/imsefacpub/10

This Article is brought to you for free and open access by the Industrial and Management Systems Engineering at DigitalCommons@University of Nebraska - Lincoln. It has been accepted for inclusion in Industrial and Management Systems Engineering Faculty Publications by an authorized administrator of DigitalCommons@University of Nebraska - Lincoln. 
Published in International Encyclopedia of Ergonomics and Human Factors, ed. Waldemar Karwowski (London \& New York: Taylor \& Francis, 2001; Second edition, Boca Raton, FL: CRC Press, 2006), pp. 3093-3096. Copyright @ 2001, 2006 by Taylor \& Francis Group, LLC. Used by permission.

\title{
Digital Human Models for Ergonomics
}

\author{
J. C. Woldstad \\ Texas Tech University, Lubbock, TX 79409-3061, USA
}

\section{Introduction}

Digital human models in the context of this section are computer-generated representations of human beings used in computer-aided design (CAD) or similar programs. These models are increasingly being used by ergonomists and other engineers to design both equipment and work environments to meet the needs of human operators. They have the advantage of allowing the designer to explore the potential advantages and disadvantages of different design configurations without requiring the construction of expensive physical mockups used in the past.

Using a digital human model, design engineers can position and manipulate operators of varying anthropometry within the simulated work environment. A variety of different analyses can be performed depending on the sophistication of the computer package. Currently available analysis modules include: reach and clearances, field of vision and visual obstructions, work posture and biomechanics, metabolic energy expenditure, time and motion, and others. In addition, the ability of these models to dynamically simulate human operators within proposed engineering projects has become a popular presentation and sales tools.

\section{Model Structure and Form}

Most digital human models are composed of an underlying rigid-link framework similar to that used in many biomechanical models of the human body. This framework assumes that the body can be represented as a series of straight mechanical links connected at pin joints that roughly corresponds to the human skeleton. The sophistication of this framework will vary considerably from a relatively simple representation (Figure 1) to models that include more than 90 different links and 140 degrees of freedom. In addition, more sophisticated geometric models of the skeleton have been developed for joints that cannot be easily modeled using a rigid link structure. Most notably, a spherical model of the shoulder joint has been developed in an attempt to model the complexities of the shoulder complex (Badler et al. 1993).
The underlying skeletal structure of digital human models is almost always covered with solid geometric constructs used to represent the surface of the human body. Initially, these constructs or geometric primitives consisted of articulated polygons that would leave noticeable gaps between the segments when the model was positioned in extended postures. More recently, complex geometric models and smoothing algorithms have been developed that virtually eliminate the robot-like appearance of some of these models. In addition to surface geometry to represent the human body, most models come with a variety of different clothing options that can be applied to the model. Clothing options often interact with range-of-motion and collision detection components of the model to increase the realism.

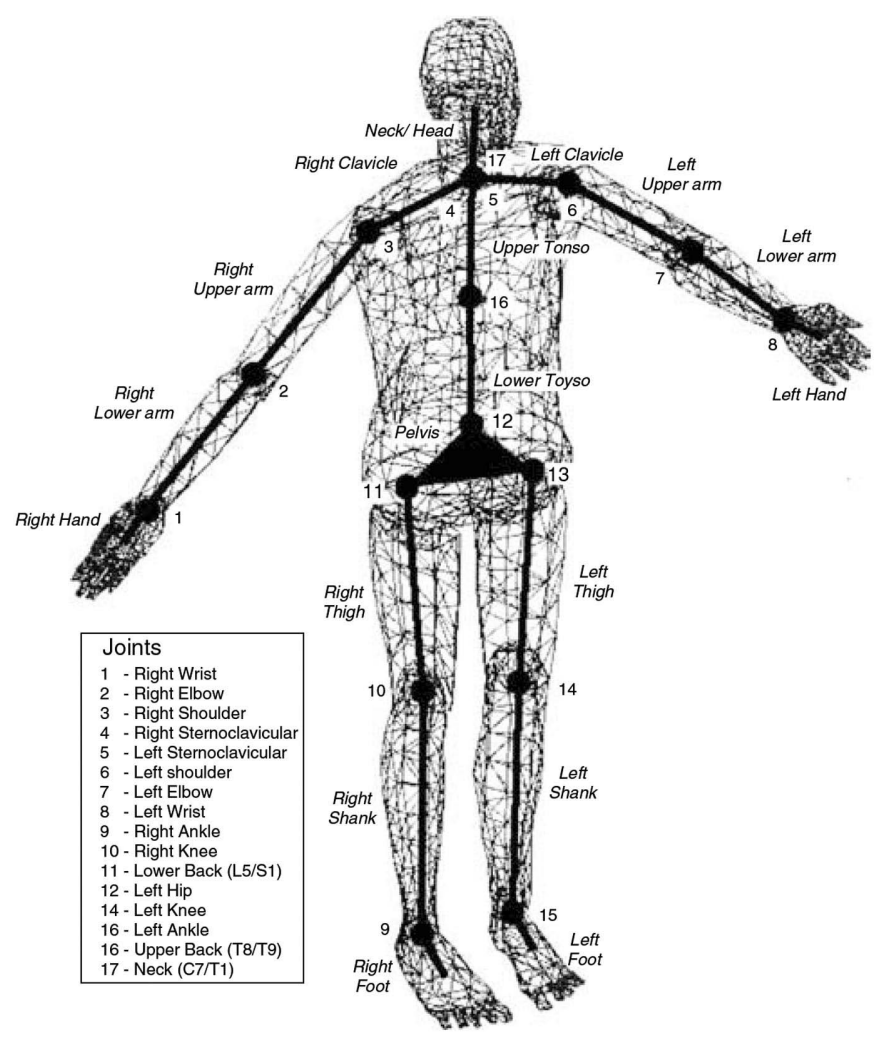

Figure 1. Typical simple three-dimensional link system for the human body. 


\section{Anthropometry, Posture, and Motion}

Anthropometric dimensions for most models can either be inputted directly or extracted from a number of existing databases. A fundamental limitation of digital human models is their inability to capture the large variation in human size and shape. Designers often desire models or manikins representative of various percentiles of the population (usually the 5th percentile female, 95th percentile male). Unfortunately, manikins of this type cannot be accurately constructed due to the inherent co-variance between human dimensions. The designers of digital human models have derived a number of different solutions to overcome this limitation. One approach has been to statistically model the relationships between different dimensions and then to select multiple dimensions that represent the boundary of a combined or joint confidence interval. Another has been to construct a family of representative anthropometries for the population under study. This group of manikins is either selected using Monte Carlo techniques or using data for whole-body surface scans of actual individuals.

To be effectively used, manikins not only need to be dimensioned, but they must be accurately positioned in the work environment. Similar to the anthropometric modules, most models allow the user to manipulate the posture of the manikin directly or use postures generated by the computer program. Postures generated by the program can be from a library of predefined "typical" human postures for the desired activity or generated using an inverse kinematics algorithm. Postures in most programs are limited by the known range-of-motion for the joint being positioned. Range-of-motion data is based on several anthropometric surveys that have collected this information in addition to body dimensions. Accurately reproducing human body postures in a simulated environment has proven to be one of the more difficult tasks in constructing digital human models. As noted above, many human models have a large number of link segments and joints requiring the specification of many different parameters to fully specify the posture (some models have more than 140 degrees of freedom). Human body postures in general are also highly indeterminate or redundant, meaning that when the position of the hands and feet are fully specified, the rest of the body can still be positioned in many different ways. Research to understand how the human body selects from the many different potential postures available is currently ongoing at several universities and has not yet produced definitive results.

Human body motion algorithms are also included with many computer packages. These can produce typical reach, lift, and grasp motions for a stationary model, or walking and carrying motions for a model moving through the environment. Problems accurately predicting human motion are analogous to those associated with predicting postures, with even a higher level com- plexity involved. Similarly, our current understanding of human motion is not sufficient to allow truly accurate representation in a virtual environment. However, the algorithms employed often produce visually compelling animations of work activities and environments.

\section{Reaches and Clearances}

The principle use of digital human models by designers has been the comparison of the dimensions and limitations of the human body to the geometry of the proposed device or work area. Work envelopes can be easily calculated using these models to indicate the portion of the environment that can easily be reached by a worker in the simulated environment. Similarly, collision detection components to most models can be used to determine when and how a worker's body will come in contact with a component of the environment so that clearances can be determined. The determination of reaches and clearances depend upon the anthropometry of the individual manikin employed and the use of many different anthropometries that are representative of the population of interest (as discussed above) is usually necessary for accurate estimates. In addition, the posture and motion components to a model can play a key role in obtaining accurate estimates. Figure 2 below

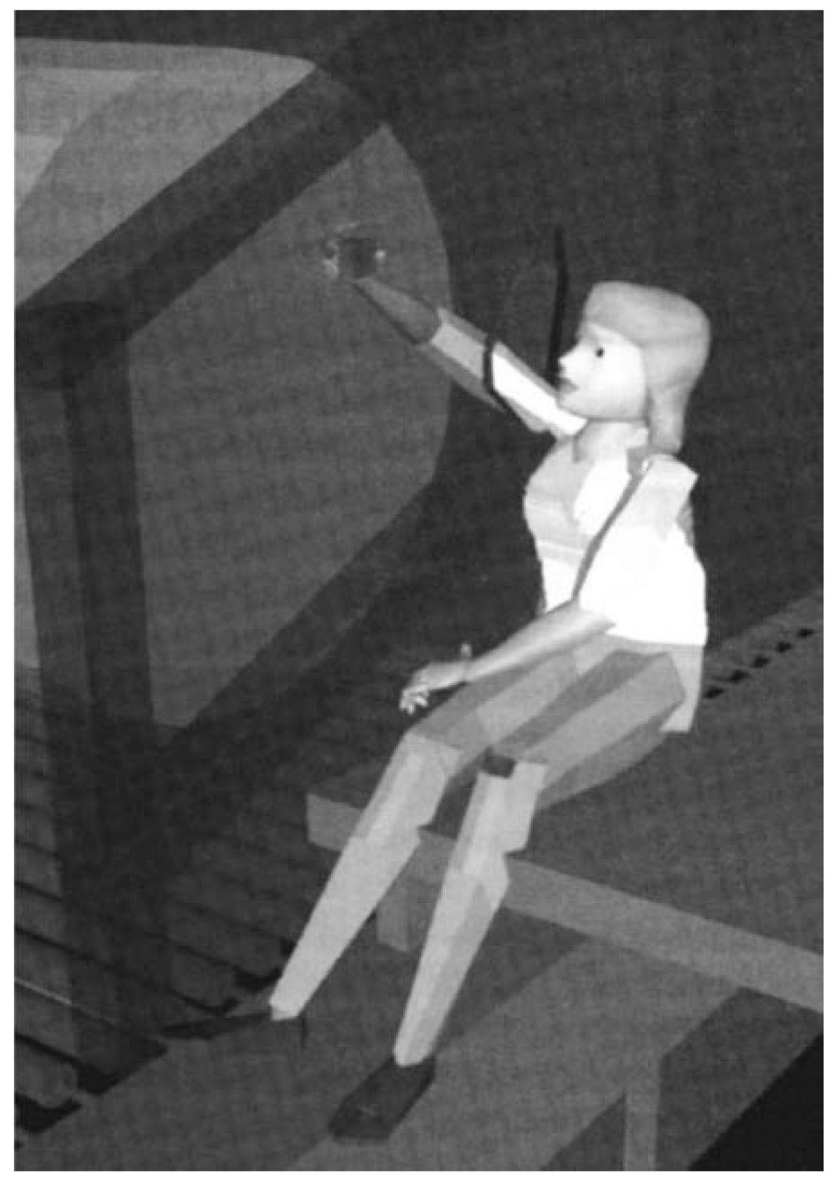

Figure 2. Reach and clearance evaluation (reprinted with permission from Engineering Animation, Inc., 1999). 
shows a typical use of a digital human model to determine both a reach and a clearance. In Figure 2, the operator must insert her arm into a limiting enclosure to manipulate a tool or device. The model is able to show how the enclosure limits the reach, and to some extent the motion of the operator.

\section{Vision}

Several models also allow the user to view the virtual environment from the perspective of the human manikin within the environment. This view allows the designer

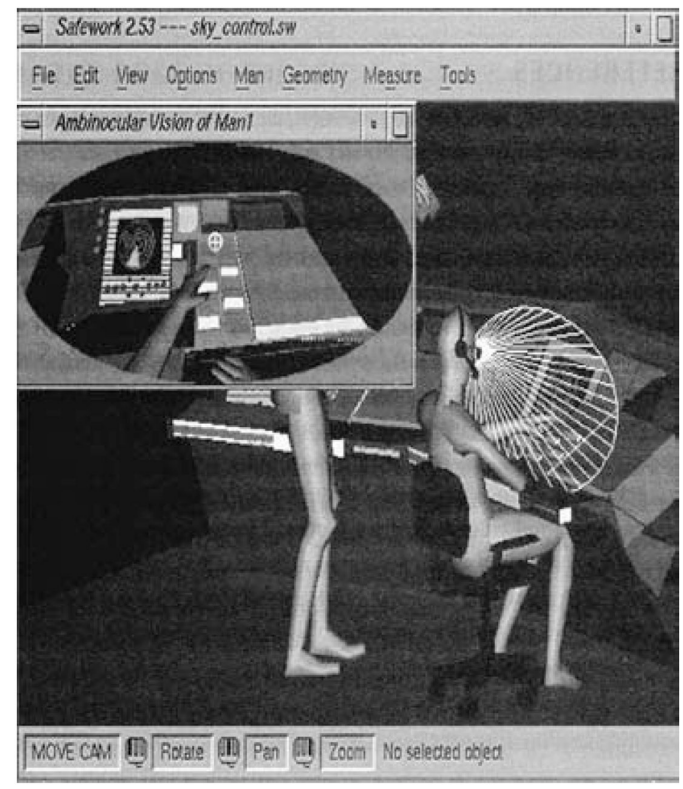

Figure 3. Field-of-view evaluation (reprinted with permission from Safework, Inc. (1999)). to explore the environment for potential visual obstructions. The view generated depends on the anthropometry, posture, and motion of the manikin, as well as, assumptions regarding the useful field of human vision. Figure 3 shows a typical scene from the point of view of a manikin within a virtual work environment. In this case the position of displays and controls for the seated operator are of concern in the design.

\section{Biomechanics}

Biomechanical models are often incorporated into modeling packages to allow the designer to estimate the mechanical stresses placed upon the internal structures of body during different activities. The objective is to estimate how work activities stress the bones, muscles and connective tissues of the body and to predict when these stresses will lead to damage of these structures. This approach is very popular in ergonomics because it closely corresponds with most expert views of the etiology of injury during manual materials handling (NIOSH 1981). Simple biomechanical models estimate the torque placed upon the joints of the body related to a work activity. More complex models will estimate parameters such as joint strength capabilities, internal muscle forces, and lower back intervertebral disc compressive force. A majority of models currently used are either based on or are similar to the 3D Static Strength Prediction Program produced by the University of Michigan (Chaffin et al. 1999). A typical output from this program is shown in Figure 4.

Biomechanical models are either two- or three- dimensional and either static or dynamic. For static mod-
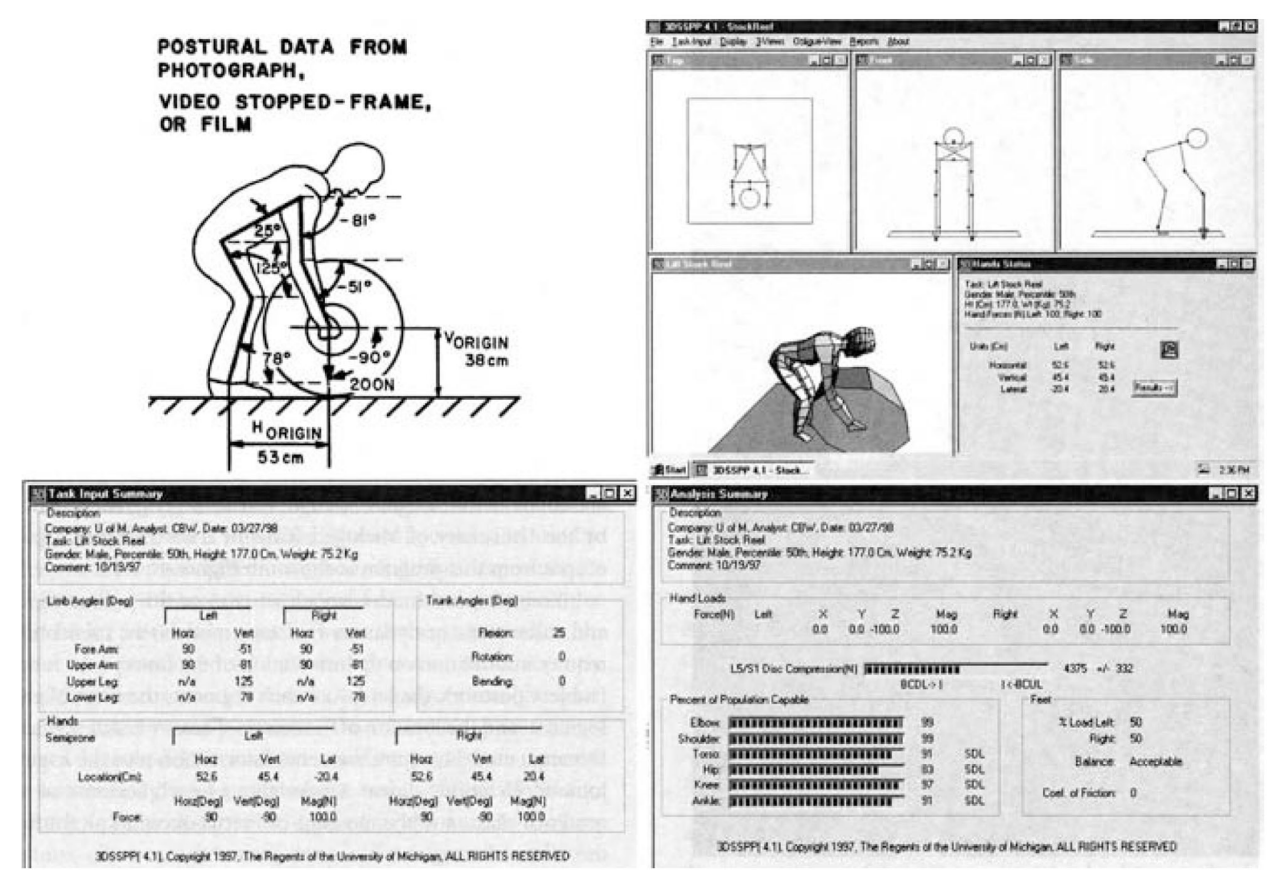

Figure 4. Work task and main output screens for the University of Michigan 3D Static Strength Prediction Program ${ }^{\text {TM }}$ (reprinted with permission from the University of Michigan (1999)). 
els, the calculations require information on the orientation of the links in the model (subject posture), the length of each segment, the mass of each segment, and the location of the center-of-mass or each segment. Dynamic models require this same information plus the angular joint accelerations, linear acceleration of each segment at the center-of-mass, and the moment-of-inertia of each link through the center of mass.

\section{Other Model Features}

A variety of other model analysis tools and features in addition to those described above are available for digital human modeling software, depending on the manufacturer and the intended use of the software. Some currently available features on different models include:

o Predetermined time study analysis based on the Methods Time Measurement (MTM) system.

- Work posture analysis based on the Ovako Working Posture Analysis System (OWAS) to analyze the relative discomfort of the back, arms, and legs in working postures.

- Metabolic energy expenditure rates based on the models of Garg et al. (1978).

- Analysis of manual materials handling tasks based on the National Institute of Occupational Safety and Health's (NIOSH 1994) lifting equation and the psychophysical limits recommended by Snook and Ciriello (1991).

- Upper limb analysis based on the Rapid Upper Limb Assessment (RULA) tool.

\section{Limitations of Current Model}

Current digital human models appear to be limited much more by our understanding of ergonomics and human behavior than by computer technology. Deficiencies in currently available data on three-dimensional human anthropometry and strength limit model accuracy. In addition, we do not have a clear understanding of the basic principles behind human posture and motion, as noted previously. In many cases, the methodology used by models is not clearly specified so that qualified users can judge the accuracy of the prediction due to the pro- prietary nature of many programs. Standardization of language, computer file format and program structure is also clear needed in this technology. Undoubtedly, computer models will become more accurate as ergonomic tools advance, but in the interim, designers and ergonomics should attempt fully to understand the tools being employed in these models and use appropriate professional judgment.

\section{Recommendations}

- Digital human models are a useful tool for ergonomics analysis and will become increasingly more popular in the future.

- Models are currently available to assist designers in analyzing reaches and clearances, visual limitation, biomechanics, and a variety of other task functions.

- Limitations exist in our current anthropometric and strength databases, as well as, our current understanding of human posture and motion. Professional judgment should be exercised in interpreting model results that rely on this information.

- Additional information on human digital modeling can be obtained from the Society of Automotive Engineers (SAE), Technical Subcommittee G-13 on Human Modeling Technology.

\section{References}

Badler, N. I., Phillips, C. B., and Webber, B. L., 1993, Simulating Humans: Computer Graphics Animation and Control (New York: Oxford).

Chaffin, D. B., Andersson, G. B. J., and Martin, B. J., 1999, Occupational Biomechanics (3rd ed.) (New York: Wiley).

Garg, A., Chaffin, D. B., and Herrin, G., 1978, Prediction of metabolic rates for manual material handling jobs. American Industrial Hygiene Association Journal, 39, 661-74.

NIOSH, 1981, Work Practices Guide for Manual Lifting. NIOSH Technical Report No. 81-122 (Cincinnati: US Department of Health and Human Services, National Institute for Occupational Safety and Health).

NIOSH, 1994, Applications Manual for the Revised NIOSH Lifting Equation. NIOSH Technical Report No. 94-110 (Cincinnati: US Department of Health and Human Services, National Institute for Occupational Safety and Health).

Snook, S. H. and Ciriello, V. M., 1991, The design of manual 